\title{
Concomitants of Dual Generalized Order Statistics from Bivariate Burr III Distribution
}

\author{
Haseeb Athar*, Nayabuddin and Zuber Akhter \\ Department of Statistics \& Operations Research \\ Aligarh Muslim University, Aligarh - 202002, India \\ haseebathar@hotmail.com
}

Received 30 April 2014; Accepted 21 March 2015

\begin{abstract}
In this paper probability density function of single concomitant and joint probability density function of two concomitants of dual generalized order statistics from bivariate Burr III distribution are obtained and expressions for single and product moments are derived. Further, results are deduced for the order statistics and lower record values.
\end{abstract}

Keywords: Dual generalized order statistics; order statistics; lower record values; concomitants; bivariate Burr III distribution.

2000 Mathematics Subject Classification: 62G30.

\section{Introduction}

The probability density function $(p d f)$ of bivariate Burr III distribution [Rodriguez, 1982] is given as

$$
\begin{array}{r}
f(x, y)=c(c+1) \alpha_{1} k_{1} x^{-\left(k_{1}+1\right)} \alpha_{2} k_{2} y^{-\left(k_{2}+1\right)}\left(1+\alpha_{1} x^{-k_{1}}+\alpha_{2} y^{-k_{2}}\right)^{-(c+2)}, \\
x, y>0, \quad k_{1}, k_{2}, c, \alpha_{1}, \alpha_{2}>0
\end{array}
$$

and the corresponding distribution function $(d f)$ is

$$
F(x, y)=\left(1+\alpha_{1} x^{-k_{1}}+\alpha_{2} y^{-k_{2}}\right)^{-c}, x, y>0, \quad k_{1}, k_{2}, c, \alpha_{1}, \alpha_{2}>0 .
$$

The conditional $p d f$ of $Y$ given $X$ is

$$
f(y \mid x)=\frac{(c+1) \alpha_{2} k_{2} y^{-\left(k_{2}+1\right)}\left(1+\alpha_{1} x^{-k_{1}}\right)^{(c+1)}}{\left(1+\alpha_{1} x^{-k_{1}}+\alpha_{2} y^{-k_{2}}\right)^{(c+2)}}, y>0 .
$$

The marginal $p d f$ of $X$ is

$$
f(x)=\frac{c \alpha_{1} k_{1} x^{-\left(k_{1}+1\right)}}{\left\{1+\alpha_{1} x^{-k_{1}}\right\}^{(c+1)}}, x>0
$$

The marginal $d f$ of $X$ is

${ }^{*}$ Corresponding author 


$$
F(x)=\left(1+\alpha_{1} x^{-k_{1}}\right)^{-c}, x>0 .
$$

Order statistics, record values and several other model of ordered random variables can be viewed as special case of generalized order statistics (gos)[Kamps, 1995]. Burkschat (2003) introduced the concept of dual generalized order statistics (dgos) to enable a common approach to descending ordered $r v$ 's like reverse order statistics and lower record values.

Let $X$ be a continuous random variables with $d f F(x)$ and the $p d f f(x), x \in(\alpha, \beta)$. Further, Let $n \in N, k \geq 1, \tilde{m}=\left(m_{1}, m_{2}, \ldots, m_{\mathrm{n}-1}\right) \in \mathfrak{R}^{n-1}, M_{r}=\sum_{j=r}^{n-1} m_{j}$ such that $\gamma_{r}=k+(n-r)+M_{r} \geq 1$ for all $r \in 1,2, \ldots, n-1$. Then $X_{d}(r, n, \tilde{m}, k) r=1,2, \ldots, n$ are called $d g o s$ if their $p d f$ is given by [Burkschat et al. (2003)]

$$
k\left(\prod_{j=1}^{n-1} \gamma_{j}\right)\left(\prod_{i=1}^{n-1}\left[F\left(x_{i}\right)\right]^{m_{i}} f\left(x_{i}\right)\right)\left[F\left(x_{n}\right)\right]^{k-1} f\left(x_{n}\right)
$$

on the cone $F^{-1}(1)>x_{1} \geq x_{2} \geq \ldots \geq x_{n}>F^{-1}(0)$.

If $m_{i}=0, i=1,2, \ldots, n, k=1$, then $X_{d}(r, n, m, k)$ reduces to the $(n-r+1)$ th order statistics, $X_{n-r+1: n}$ from the sample $X_{1}, X_{2}, \ldots, X_{n}$ and at $m=-1, X_{d}(r, n, m, k)$ reduces to $r$ th, $k$ - lower record values. For more details on order statistics and record values, one may refer to David and Nagaraja (2003) and Ahsanullah (2004) respectively.

In view of (1.6), the $p d f$ of $X_{d}(r, n, m, k)$ is

$$
f_{r, n, m, k}(x)=\frac{C_{r-1}}{(r-1) !}[F(x)]^{\gamma_{r}-1} f(x) g_{m}^{r-1}(F(x))
$$

and joint $p d f$ of $X_{d}(r, n, m, k)$ and $X_{d}(s, n, m, k), 1 \leq r<s \leq n$ is

$$
\begin{gathered}
f_{r, s, n, m, k}(x, y)=\frac{C_{s-1}}{(r-1) !(s-r-1) !}[F(x)]^{m} f(x) g_{m}^{r-1}(F(x)) \\
\times\left[h_{m}(F(y))-h_{m}(F(x))\right]^{s-r-1}[F(y)]^{\gamma_{s}-1} f(y), x>y
\end{gathered}
$$

where

$$
\begin{aligned}
& C_{r-1}=\prod_{i=1}^{r} \gamma_{i} \\
& h_{m}(x)= \begin{cases}-\frac{1}{m+1} x^{m+1} & , m \neq-1 \\
-\log x & , m=-1\end{cases}
\end{aligned}
$$

and

$$
g_{m}(x)=h_{m}(x)-h_{m}(1), x \in(0,1) .
$$

Let $\left(X_{i}, Y_{i}\right), i=1,2, \ldots, n$, be the $n$ pairs of independent random variables from some bivariate population with distribution function $F(x, y)$. If we arrange the $X$ - variates in descending order as 
$X(1, n, m, k) \geq X(2, n, m, k) \geq \ldots \geq X(n, n, m, k)$ then $Y$ - variates paired (not necessarily in descending order ) with these dual generalized ordered statistics are called the concomitants of dual generalized order statistics and are denoted by $Y_{[1, n, m, k]}, Y_{[2, n, m, k]}, \ldots, Y_{[n, n, m, k]}$. The $p d f$ of $Y_{[r, n, m, k]}$, the $r-t h$ concomitant of dual generalized order statistics is given as

$$
g_{[r, n, m, k]}(y)=\int_{-\infty}^{\infty} f(y \mid x) f_{r, n, m, k}(x) d x
$$

and the joint $p d f$ of $Y_{[r, n, m, k]}$ and $Y_{[s, n, m, k]}, 1 \leq r<s \leq n$ is

$$
g_{[r, s, n, m, k]}\left(y_{1}, y_{2}\right)=\int_{-\infty}^{\infty} \int_{-\infty}^{x_{1}} f\left(y_{1} \mid x_{1}\right) f\left(y_{2} \mid x_{2}\right) f_{r, s, n, m, k}\left(x_{1}, x_{2}\right) d x_{2} d x_{1} .
$$

\section{Probability Density Function of $Y_{[r, n, m, k]}$}

For the bivariate Burr III distribution as given in (1.1), the $p d f$ of $g_{[r, n, m, k]}(y)$ in view of (1.3), (1.4), (1.5), (1.7) and (1.9) is given as

$$
\begin{aligned}
g_{[r, n, m, k]}(y)= & \frac{\alpha_{2} k_{2} C_{r-1}}{(r-1) !(m+1)^{r-1}} c(c+1) y^{-\left(1+k_{2}\right)} \sum_{i=0}^{r-1}(-1)^{i}\left(\begin{array}{c}
r-1 \\
i
\end{array}\right) \\
& \times \int_{0}^{\infty} \frac{\alpha_{1} k_{1} x^{-\left(1+k_{1}\right)}}{\left(1+\alpha_{1} x^{-k_{1}}+\alpha_{2} y^{-k_{2}}\right)^{c+2}} \frac{1}{\left(1+\alpha_{1} x^{-k_{1}}\right)^{c\left(\gamma_{r-i}-1\right)}} d x .
\end{aligned}
$$

Let $t=\alpha_{1} x^{-k_{1}}$, then the R.H.S. of (2.1) reduces to

$$
\begin{aligned}
& =\frac{\alpha_{2} k_{2} C_{r-1}}{(r-1) !(m+1)^{r-1}} c(c+1) y^{-\left(1+k_{2}\right)} \sum_{i=0}^{r-1}(-1)^{i}\left(\begin{array}{c}
r-1 \\
i
\end{array}\right) \\
& \times \int_{0}^{\infty}\left(1+t+\alpha_{2} y^{-k_{2}}\right)^{-(c+2)}(1+t)^{-c\left(\gamma_{r-i}-1\right)} d t .
\end{aligned}
$$

Since

$$
\begin{array}{r}
\left.\int_{0}^{\infty} x^{v-1}(a+x)^{-\mu}(x+y)^{-\rho} d x=\frac{\Gamma v \Gamma(\mu-v+\rho)}{\Gamma(\mu+\rho) a^{\mu}} y^{(v-\rho)}{ }_{2} F_{1}\left[\begin{array}{r}
\mu, \\
v+\rho \\
\mu+\rho
\end{array}\right] 1-\frac{y}{a}\right] \\
{[|\arg a|<\Pi, \operatorname{Re} v>0,|\arg y|<\Pi, \operatorname{Re} \rho>\operatorname{Re}(v-\mu)],}
\end{array}
$$

where

$$
{ }_{2} F_{1}\left[\begin{array}{l}
a, b \\
c
\end{array}\right]=\sum_{p=0}^{\infty} \frac{(a)_{p}(b)_{p} x^{p}}{(c)_{p} p !}
$$

is the Gauss hypergeometric series

$$
(\lambda)_{n}=\frac{\Gamma(\lambda+n)}{\Gamma \lambda}, \lambda \neq 0,-1,-2, \ldots, \quad[\text { Prudnikov et. al }, 1986]
$$

is the Pochhammer symbol . 
Haseeb Athar, Nayabuddin and Zuber Akhter

Therefore (2.2), becomes

$$
\begin{aligned}
& g_{[r, n, m, k]}(y)=\frac{\alpha_{2} k_{2} C_{r-1}}{(r-1) !(m+1)^{r-1}} c(c+1) y^{-\left(1+k_{2}\right)} \sum_{i=0}^{r-1}(-1)^{i}\left(\begin{array}{c}
r-1 \\
i
\end{array}\right) \\
& \times \frac{\left(1+\alpha_{2} y^{-k_{2}}\right)^{-(c+1)}}{\left(c \gamma_{r-i}+1\right)}{ }_{2} F_{1}\left[\begin{array}{l}
\left(c \gamma_{r-i}-c\right), 1 \\
\left(c \gamma_{r-i}+2\right)
\end{array} ;-\alpha_{2} y^{-k_{2}}\right] .
\end{aligned}
$$

\section{Moment of $Y_{[r, n, m, k]}$}

For the bivariate Burr III distribution as given in (1.1), the $a-t h$ moment of $Y_{[r, n, m, k]}$ is given as,

$$
\begin{aligned}
& E\left(Y_{[r, n, m, k]}^{(a)}\right)=\int y^{a} g_{[r, n, m, k]}(y) d y \\
& =\frac{\alpha_{2} k_{2} C_{r-1}}{(r-1) !(m+1)^{r-1}} c(c+1) \sum_{i=0}^{r-1}(-1)^{i}\left(\begin{array}{c}
r-1 \\
i
\end{array}\right) \frac{1}{c \gamma_{r-i}+1} \\
& \quad \times \int_{0}^{\infty} y^{a} y^{-\left(1+k_{2}\right)}\left(1+\alpha_{2} y^{-k_{2}}\right)^{-(c+1)}{ }_{2} F_{1}\left[\begin{array}{c}
\left(c \gamma_{r-i}-c\right), 1 \\
\left(c \gamma_{r-i}+2\right)
\end{array}-\alpha_{2} y^{-k_{2}}\right] d y \\
& =\frac{\alpha_{2} k_{2} C_{r-1}}{(r-1) !(m+1)^{r-1}} c(c+1) \sum_{i=0}^{r-1}(-1)^{i}\left(\begin{array}{c}
r-1 \\
i
\end{array}\right) \frac{1}{c \gamma_{r-i}+1} \\
& \times \sum_{p=0}^{\infty} \frac{\left(c \gamma_{r-i}-c\right)_{p}(1)_{p}}{\left(c \gamma_{r-i}+2\right)_{p} p !} \int_{0}^{\infty} y^{a} y^{-\left(1+k_{2}\right)}\left(1+\alpha_{2} y^{-k_{2}}\right)^{-(c+1)}\left(-\alpha_{2} y^{-k_{2}}\right)^{p} d y .
\end{aligned}
$$

Now letting $t=1+\alpha_{2} y^{-k_{2}}$ in (3.3), we get

$$
\begin{aligned}
= & \frac{\left(\alpha_{2}\right)^{\frac{a}{k_{2}}} C_{r-1}}{(r-1) !(m+1)^{r-1}} c(c+1) \sum_{i=0}^{r-1}(-1)^{i}\left(\begin{array}{c}
r-1 \\
i
\end{array}\right) \frac{1}{c \gamma_{r-i}+1} \\
& \times \sum_{p=0}^{\infty} \frac{\left(c \gamma_{r-i}-c\right)_{p}(1)_{p}(-1)^{p}}{\left(c \gamma_{r-i}+2\right)_{p} p !} \int_{1}^{\infty} t^{-(c+1)}(t-1)^{\left(p-\frac{a}{k_{2}}\right)} d t .
\end{aligned}
$$

Since

$$
\int_{y}^{\infty} x^{-\lambda}(x-y)^{\mu-1} d x=\frac{\Gamma(\lambda-\mu) \Gamma \mu}{\Gamma \lambda} y^{(\mu-\lambda)}, 0<\operatorname{Re} \mu<\operatorname{Re}<\lambda
$$

[c.f. Erdélyi et al., 1954]. 
Therefore R.H.S. of (3.4) becomes

$$
\begin{aligned}
& =\frac{\left(\alpha_{2}\right)^{\frac{a}{k_{2}}} C_{r-1}}{(r-1) !(m+1)^{r-1}} c(c+1) \sum_{i=0}^{r-1}(-1)^{i}\left(\begin{array}{c}
r-1 \\
i
\end{array}\right) \frac{1}{c \gamma_{r-i}+1} \\
& \times \sum_{p=0}^{\infty} \frac{\left(c \gamma_{r-i}-c\right)_{p}(1)_{p}(-1)^{p}}{\left(c \gamma_{r-i}+2\right)_{p} p !} \frac{\Gamma\left(c+\frac{a}{k_{2}}-p\right) \Gamma\left(p+1-\frac{a}{k_{2}}\right)}{\Gamma(c+1)} .
\end{aligned}
$$

Now on application of (2.5) and using

$$
\left.(\lambda)_{-n}=\frac{(-1)^{n}}{(1-\lambda)_{n}}, n=1,2,3, \ldots, \lambda \neq 0, \pm 1, \pm 2, \ldots \text { [Srivastava and Karlsson, } 1985\right]
$$

in (3.6) and simplifying, we get

$$
\begin{aligned}
E\left(Y_{[r, n, m, k]}^{(a)}\right) & =\frac{\left(\alpha_{2}\right)^{\frac{a}{k_{2}}} C_{r-1}}{(r-1) !(m+1)^{r-1}} c(c+1) \sum_{i=0}^{r-1}(-1)^{i}\left(\begin{array}{c}
r-1 \\
i
\end{array}\right) \frac{1}{c \gamma_{r-i}+1} \\
& \times \sum_{p=0}^{\infty} \frac{\left(c \gamma_{r-i}-c\right)_{p}(1)_{p}\left(1-\frac{a}{k_{2}}\right)_{p}}{\left(c \gamma_{r-i}+2\right)_{p}\left(1-c-\frac{a}{k_{2}}\right)_{p} p !} \frac{\Gamma\left(c+\frac{a}{k_{2}}\right) \Gamma\left(1-\frac{a}{k_{2}}\right)}{\Gamma(c+1)} .
\end{aligned}
$$

We have the generalized Gauss series or the generalized hypergeometric series.

$$
{ }_{p} F_{q}\left[\begin{array}{ccc}
\alpha_{1} & \ldots & \alpha_{p} \\
& & \\
\beta_{1} \ldots & \beta_{q}
\end{array}\right]=\sum_{k=0}^{\infty} \frac{\left(\alpha_{1}\right)_{k}\left(\alpha_{2}\right)_{k} \ldots\left(\alpha_{p}\right)_{k}}{\left(\beta_{1}\right)_{k}\left(\beta_{2}\right)_{k} \ldots\left(\beta_{q}\right)_{k}} \frac{z^{k}}{k !}
$$

Thus applying (3.9) in (3.8), we have

$$
\begin{aligned}
& E\left(Y_{[r, n, m, k]}^{(a)}\right)=\frac{\left(\alpha_{2}\right)^{\frac{a}{k_{2}}} C_{r-1} c(c+1)}{(r-1) !(m+1)^{r-1}} \sum_{i=0}^{r-1}(-1)^{i}\left(\begin{array}{c}
r-1 \\
i
\end{array}\right) \frac{1}{c \gamma_{r-i}+1} \frac{\Gamma\left(c+\frac{a}{k_{2}}\right) \Gamma\left(1-\frac{a}{k_{2}}\right)}{\Gamma(c+1)} \\
& \times{ }_{3} F_{2}\left[\begin{array}{ccc}
\left(c \gamma_{r-i}-c\right), & 1, & \left(1-\frac{a}{k_{2}}\right) \\
\left(c \gamma_{r-i}+2\right), & \left(1-c-\frac{a}{k_{2}}\right) & , 1
\end{array}\right] .
\end{aligned}
$$

Noting that [prudnikov et al, 1986] 


$$
\begin{aligned}
& { }_{3} F_{2}\left[\begin{array}{ccc}
-N, & 1, & a \\
b, & a-m, & , 1
\end{array}\right] \\
& =\frac{(b-1)(a-m-1)}{(b+N-1)(a-1)} 3 F_{2}\left[\begin{array}{ccc}
-m, & 1, & 2-b \\
2-b-N, & 2-a,
\end{array}\right] .
\end{aligned}
$$

Now using (3.11) in (3.10), we can write

$$
\begin{aligned}
& =\frac{\left(\alpha_{2}\right)^{\frac{a}{k_{2}}} c C_{r-1}}{(r-1) !(m+1)^{r-1}} \sum_{i=0}^{r-1}(-1)^{i}\left(\begin{array}{c}
r-1 \\
i
\end{array}\right) \frac{\Gamma\left(c+\frac{a}{k_{2}}\right) \Gamma\left(1-\frac{a}{k_{2}}\right)}{\Gamma(c+1)} \frac{\left(c+\frac{a}{k_{2}}\right)}{\left(\frac{a}{k_{2}}\right)} \\
& \times{ }_{3} F_{2}\left[\begin{array}{llll}
-c, & 1, & \left(-c \gamma_{r-i}\right) & \\
-c, 1+\frac{a}{k_{2}}, & , 1
\end{array}\right] .
\end{aligned}
$$

Using relation (3.9) in (3.12), we get

$$
\begin{aligned}
& =\frac{\left(\alpha_{2}\right)^{\frac{a}{k_{2}}} c C_{r-1}}{(r-1) !(m+1)^{r-1}} \sum_{i=0}^{r-1}(-1)^{i}\left(\begin{array}{c}
r-1 \\
i
\end{array}\right) \frac{\Gamma\left(c+\frac{a}{k_{2}}\right) \Gamma\left(1-\frac{a}{k_{2}}\right)}{\Gamma(c+1)} \frac{\left(c+\frac{a}{k_{2}}\right)}{\left(\frac{a}{k_{2}}\right)} \\
& \times{ }_{2} F_{1}\left[\begin{array}{c}
1, \quad\left(-c \gamma_{r-i}\right) \\
\left(1+\frac{a}{k_{2}}\right)
\end{array}\right] .
\end{aligned}
$$

Noting that [Prudnikov et al., 1986].

$$
{ }_{2} F_{1}\left[\begin{array}{l}
a b \\
c
\end{array} ; 1\right]=\frac{\Gamma(c) \Gamma(c-a-b)}{\Gamma(c-a) \Gamma(c-b)}, \operatorname{Re}(c-a-b)>0, c \neq, \pm 1, \pm 2, \ldots
$$

Applying (3.14) in (3.13), we get 


$$
\begin{aligned}
& =\frac{\left(\alpha_{2}\right)^{\frac{a}{k_{2}}} C_{r-1}}{(r-1) !(m+1)^{r-1}} \sum_{i=0}^{r-1}(-1)^{i}\left(\begin{array}{c}
r-1 \\
i
\end{array}\right) \frac{\left(c+\frac{a}{k_{2}}\right) \Gamma\left(c+\frac{a}{k_{2}}\right) \Gamma\left(1-\frac{a}{k_{2}}\right)}{c \Gamma(c)} \frac{1}{\gamma_{r-i}+\frac{a}{c k_{2}}} \\
& =\frac{\left(\alpha_{2}\right)^{\frac{a}{k_{2}}} C_{r-1}}{(r-1) !(m+1)^{r}} \sum_{i=0}^{r-1}(-1)^{i}\left(\begin{array}{c}
r-1 \\
i
\end{array}\right) \frac{\left(c+\frac{a}{k_{2}}\right) \Gamma\left(c+\frac{a}{k_{2}}\right) \Gamma\left(1-\frac{a}{k_{2}}\right)}{\Gamma(c+1)} \\
& \quad \times B\left(\frac{k}{m+1}+(n-r)+i+\frac{a}{c k_{2}(m+1)}, 1\right) .
\end{aligned}
$$

Since,

$$
\sum_{a=0}^{b}(-1)^{a}\left(\begin{array}{l}
b \\
a
\end{array}\right) B(a+k, c)=B(k, c+b)
$$

where $B(a, b)$ is the complete beta function.

Therefore,

$$
\begin{aligned}
E\left(y_{[r, n, m, k]}^{(a)}\right) & =\frac{\left(\alpha_{2}\right)^{\frac{a}{k_{2}}} C_{r-1}}{(m+1)^{r}} \frac{\Gamma\left(c+1+\frac{a}{k_{2}}\right) \Gamma\left(1-\frac{a}{k_{2}}\right)}{c \Gamma(c)} \frac{\Gamma\left(\frac{k+(n-r)(m+1)+\frac{a}{c k_{2}}}{m+1}\right)}{\Gamma\left(\frac{k+n(m+1)+\frac{a}{c k_{2}}}{m+1}\right)} \\
E\left(y_{[r, n, m, k]}^{(a)}\right) & =\left(\alpha_{2}\right)^{\frac{a}{k_{2}}} \frac{\Gamma\left(c+1+\frac{a}{k_{2}}\right) \Gamma\left(1-\frac{a}{k_{2}}\right)}{c \Gamma(c)} \frac{1}{\prod_{i=1}^{r}\left(1+\frac{a}{c k_{2} \gamma_{i}}\right)} .
\end{aligned}
$$

Remark 3.1 : If $m=0, k=1$ in (3.11), we get single moment of concomitants of order statistics from bivariate Burr III distribution as;

$$
E\left(y_{[n-r+1: n]}^{(a)}\right)=\frac{n !}{(n-r) !}\left(\alpha_{2}\right)^{\frac{a}{k_{2}}} \frac{\Gamma\left(c+1+\frac{a}{k_{2}}\right) \Gamma\left(1-\frac{a}{k_{2}}\right)}{c \Gamma(c)} \frac{\Gamma\left(n-r+1+\frac{a}{c k_{2}}\right)}{\Gamma\left(n+1+\frac{a}{c k_{2}}\right)} .
$$

If we replace $n-r+1$ by $r$, then

$$
E\left(y_{[r: n]}^{(a)}\right)=\frac{n !}{(r-1) !}\left(\alpha_{2}\right)^{\frac{a}{k_{2}}} \frac{\Gamma\left(c+1+\frac{a}{k_{2}}\right) \Gamma\left(1-\frac{a}{k_{2}}\right)}{c \Gamma(c)} \frac{\Gamma\left(r+\frac{a}{c k_{2}}\right)}{\Gamma\left(n+1+\frac{a}{c k_{2}}\right)}
$$

and at $r=n$, we get 


$$
E\left(y_{[n: n]}^{(a)}\right)=\frac{n}{\left(n c+\frac{a}{k_{2}}\right)}\left(\alpha_{2}\right)^{\frac{a}{k_{2}}} \frac{\Gamma\left(c+1+\frac{a}{k_{2}}\right) \Gamma\left(1-\frac{a}{k_{2}}\right)}{\Gamma(c)} .
$$

It may be noted that for the order statistics, the $k^{\text {th }}$ moments of $Y_{[r: n]}$ is

$$
\mu_{[r: n]}^{k}(y)=\sum_{i=r}^{n}(-1)^{i-r}\left(\begin{array}{c}
i-1 \\
n-r
\end{array}\right)\left(\begin{array}{c}
n \\
i
\end{array}\right) \mu_{[i: i]}^{k}(y) .
$$

Therefore,

$$
E\left(y_{[r: n]}^{(a)}\right)=\sum_{i=r}^{n}(-1)^{i-r}\left(\begin{array}{c}
i-1 \\
r-1
\end{array}\right)\left(\begin{array}{c}
n \\
i
\end{array}\right) \frac{i}{\left(i c+\frac{a}{k_{2}}\right)}\left(\alpha_{2}\right)^{\frac{a}{k_{2}}} \frac{\Gamma\left(c+1+\frac{a}{k_{2}}\right) \Gamma\left(1-\frac{a}{k_{2}}\right)}{\Gamma(c)}
$$

as obtained by (Begum and Khan,1998).

Remark 3.2 : Set $m=-1$ in (3.12), to get moment of $k$-th lower record value from bivariate Burr III distribution as;

$$
E\left(y_{[r, n,-1, k]}^{(a)}\right)=\left(\alpha_{2}\right)^{\frac{a}{k_{2}}} \frac{\Gamma\left(c+1+\frac{a}{k_{2}}\right) \Gamma\left(1-\frac{a}{k_{2}}\right)}{c \Gamma(c)} \frac{1}{\left(1+\frac{a}{c k k_{2}}\right)^{r}} .
$$

\section{Joint Probability Density Function of $Y_{[r, n, m, k]}$ and $Y_{[s, n, m, k]}$}

For the bivariate Burr III distribution as given in (1.1), the joint $p d f$ of $Y_{[r, n, m, k]}$ and $Y_{[s, n, m, k]}$ in view of (1.3), (1.4), (1.5), (1.8) and (1.10) is given as

$$
\begin{aligned}
& g_{[r, s, n, m, k]}\left(y_{1}, y_{2}\right)=\frac{C_{s-1}}{(r-1) !(s-r-1) !(m+1)^{s-2}} c^{2}(c+1)^{2}\left(\alpha_{1} k_{1}\right)\left(\alpha_{2} k_{2}\right)^{2} \\
& \quad \times y_{1}^{-\left(1+k_{2}\right)} y_{2}^{-\left(1+k_{2}\right)} \sum_{i=0}^{r-1} \sum_{j=0}^{s-r-1}(-1)^{i+j}\left(\begin{array}{c}
r-1 \\
i
\end{array}\right)\left(\begin{array}{c}
s-r-1 \\
j
\end{array}\right) \\
& \quad \times \int_{0}^{\infty} \frac{x_{1}^{-\left(1+k_{1}\right)}}{\left(1+\alpha_{1} x_{1}^{-k_{1}}\right)^{c(s-r+i-j)(m+1)-c}} \frac{1}{\left(1+\alpha_{1} x_{1}^{-k_{1}}+\alpha_{2} y_{1}^{-k_{2}}\right)^{c+2}} I\left(x_{1}, y_{2}\right) d x_{1}
\end{aligned}
$$

where,

$$
I\left(x_{1}, y_{2}\right)=\int_{0}^{x_{1}} \frac{\alpha_{1} k_{1} x_{2}^{-\left(1+k_{1}\right)}}{\left(1+\alpha_{1} x_{2}^{-k_{1}}\right)^{c\left(\gamma_{s-j}-1\right)}} \frac{1}{\left(1+\alpha_{1} x_{2}^{-k_{1}}+\alpha_{2} y_{2}^{-k_{2}}\right)^{c+2}} d x_{2} .
$$

Setting $t_{2}=\left(1+\alpha_{1} x_{2}^{-k_{1}}\right)$, then the R.H.S. of (4.2) reduces to 


$$
I\left(x_{1}, y_{2}\right)=(\lambda)^{-\beta} \int_{1+\alpha_{1} x_{2}^{-k_{1}}}^{\infty} t_{2}^{-\alpha}\left(1+\frac{t_{2}}{\lambda}\right)^{-\beta} d t_{2}
$$

where $\alpha=\left(c \gamma_{s-j}-c\right), \beta=(c+2)$ and $\lambda=\alpha_{2} y_{2}^{-k_{2}}$

Note that [Prudnikov et al., 1986].

$$
(1+z)^{-a}=\sum_{p=0}^{\infty} \frac{(-1)^{p}(a)_{p} z^{p}}{p !}
$$

Now in view of (4.4), (4.3) after simplification yields

$$
I\left(x_{1}, y_{2}\right)=(\lambda)^{-\beta} \sum_{l=0}^{\infty}(-1)^{l} \frac{(\beta)_{l}\left(\frac{1}{\lambda}\right)^{l}}{l !} \frac{\left(1+\alpha_{1} x_{1}^{-k_{1}}\right)^{-(\alpha-l-1)}}{(\alpha-l-1)} .
$$

Now putting the value of $I\left(x_{1}, y_{2}\right)$ from (4.5) in (4.1), we obtain

$$
\begin{aligned}
& g_{[r, s, n, m, k]}\left(y_{1}, y_{2}\right)=\frac{c^{2}(c+1)^{2}\left(\alpha_{1} k_{1}\right)\left(\alpha_{2} k_{2}\right)^{2} C_{s-1}}{(r-1) !(s-r-1) !(m+1)^{s-2}} y_{1}^{-\left(1+k_{2}\right)} y_{2}^{-\left(1+k_{2}\right)} \\
& \quad \times \sum_{i=0}^{r-1} \sum_{j=0}^{s-r-1}(-1)^{i+j}\left(\begin{array}{c}
r-1 \\
i
\end{array}\right)\left(\begin{array}{c}
s-r-1 \\
j
\end{array}\right)(\lambda)^{-\beta} \sum_{l=0}^{\infty} \frac{(-1)^{l}}{(\alpha-l-1)} \frac{(\beta)_{l}\left(\frac{1}{\lambda}\right)^{l}}{l !} \\
& \times \int_{0}^{\infty} \frac{x_{1}^{-\left(1+k_{1}\right)}}{\left(1+\alpha_{1} x_{1}^{-k_{1}}\right)^{c(s-r+i-j)(m+1)-c+\left(c \gamma_{s-j}-c\right)-l-1}} \frac{1}{\left(1+\alpha_{1} x_{1}^{-k_{1}}+\alpha_{2} y_{1}^{-k_{2}}\right)^{c+2}} d x_{1} .
\end{aligned}
$$

Setting $t_{1}=\left(1+\alpha_{1} x_{1}^{-k_{1}}\right)$ in (4.6), and after simplification we get

$$
\begin{gathered}
g_{[r, s, n, m, k]}\left(y_{1}, y_{2}\right)=\frac{C_{s-1}}{(r-1) !(s-r-1) !(m+1)^{s-2}} c^{2}(c+1)^{2}\left(\alpha_{2} k_{2}\right)^{2} \\
\times y_{1}^{-\left(1+k_{2}\right)} y_{2}^{-\left(1+k_{2}\right)} \sum_{i=0}^{r-1} \sum_{j=0}^{s-r-1}(-1)^{i+j}\left(\begin{array}{c}
r-1 \\
i
\end{array}\right)\left(\begin{array}{c}
s-r-1 \\
j
\end{array}\right) \\
\times(\lambda)^{-\beta}(\delta)^{-\beta} \sum_{l=0}^{\infty} \frac{(\beta)_{l}\left(-\frac{1}{\lambda}\right)^{l}}{(1-\alpha+l) l !} \sum_{p=0}^{\infty} \frac{(\beta)_{p}\left(-\frac{1}{\delta}\right)^{p}}{(2-\theta-\alpha+l+p) p !}
\end{gathered}
$$

where $\delta=\alpha_{2} y_{1}^{-k_{2}}$ and $\theta=c(s-r+i-j)(m+1)-c$. 
Haseeb Athar, Nayabuddin and Zuber Akhter

By setting $d=1-\alpha$ and $g=2-\theta-\alpha$ in (4.7), we get

$$
\begin{gathered}
=\frac{c^{2}(c+1)^{2}\left(\alpha_{2} k_{2}\right)^{2} C_{s-1}}{(r-1) !(s-r-1) !(m+1)^{s-2}} \sum_{i=0}^{r-1} \sum_{j=0}^{s-r-1}(-1)^{i+j}\left(\begin{array}{c}
r-1 \\
i
\end{array}\right)\left(\begin{array}{c}
s-r-1 \\
j
\end{array}\right) \\
\times y_{1}^{-\left(1+k_{2}\right)} y_{2}^{-\left(1+k_{2}\right)}(\lambda)^{-\beta}(\delta)^{-\beta} \sum_{l=0}^{\infty} \frac{(\beta)_{l}\left(-\frac{1}{\lambda}\right)^{l}}{(d+l) l !} \sum_{p=0}^{\infty} \frac{(\beta)_{p}\left(-\frac{1}{\delta}\right)^{p}}{(g+p+l) p !} .
\end{gathered}
$$

Now after putting the value of $\lambda$ and $\delta$ in (4.8), we have

$$
\begin{gathered}
=\frac{c^{2}(c+1)^{2}\left(\alpha_{2} k_{2}\right)^{2} C_{s-1}}{(r-1) !(s-r-1) !(m+1)^{s-2}} \sum_{i=0}^{r-1} \sum_{j=0}^{s-r-1}(-1)^{i+j}\left(\begin{array}{c}
r-1 \\
i
\end{array}\right)\left(\begin{array}{c}
s-r-1 \\
j
\end{array}\right) y_{1}^{-\left(1+k_{2}\right)} \\
\quad \times y_{2}^{-\left(1+k_{2}\right)}\left(\alpha_{2} y_{2}^{-k_{2}}\right)^{-\beta}\left(\alpha_{2} y_{1}^{-k_{2}}\right)^{-\beta} \sum_{l=0}^{\infty} \frac{(\beta)_{l}\left(\frac{-1}{\alpha_{2} y_{2}^{-k_{2}}}\right)^{l}}{(d+l) l !} \sum_{p=0}^{\infty} \frac{(\beta)_{p}\left(\frac{-1}{\alpha_{2} y_{1}^{-k_{2}}}\right)^{p}}{(g+p+l) p !}
\end{gathered}
$$

Noting that [Srivastava and Karlsson, 1985].

$$
\begin{aligned}
& (\lambda+m)=\frac{\lambda(\lambda+1)_{m}}{(\lambda)_{m}} \\
& (\lambda+m+n)=\frac{\lambda(\lambda+1)_{m+n}}{(\lambda)_{m+n}} .
\end{aligned}
$$

Using relation (4.10) and (4.11) in (4.9), it becomes

$$
\begin{aligned}
& =\frac{c^{2}(c+1)^{2} C_{s-1}}{(r-1) !(s-r-1) !(m+1)^{s-2}} \sum_{i=0}^{r-1} \sum_{j=0}^{s-r-1}(-1)^{i+j}\left(\begin{array}{c}
r-1 \\
i
\end{array}\right)\left(\begin{array}{c}
s-r-1 \\
j
\end{array}\right) \frac{1}{d g} \\
& \times \frac{\left(\alpha_{2} k_{2} y_{1}^{-\left(1+k_{2}\right)}\right)}{\left(\alpha_{2} y_{1}^{-k_{2}}\right)^{\beta}} \frac{\left(\alpha_{2} k_{2} y_{2}^{-\left(1+k_{2}\right)}\right)}{\left(\alpha_{2} y_{2}^{-k_{2}}\right)^{\beta}} \sum_{l=0}^{\infty} \sum_{p=0}^{\infty} \frac{(g)_{p+l}}{(g+1)_{p+l}} \frac{(\beta)_{l}(d)_{l}(\beta)_{p}}{(d+1)_{l}} \frac{\left(\frac{-1}{\alpha_{2} y_{1}^{-k_{2}}}\right)^{p}}{p !} \frac{\left(\frac{-1}{\alpha_{2} y_{2}^{-k_{2}}}\right)^{l}}{l !} .
\end{aligned}
$$

We have the Kampede Feriet's series [Srivastava and Karlsson, 1985]

$$
\begin{aligned}
& \left.F_{l: m ; n}^{p: q ; k}\left[\begin{array}{ccc}
\left(a_{p}\right):\left(b_{q}\right) ;\left(c_{k}\right) & \\
\left(\alpha_{l}\right) & \left(\beta_{m}\right)\left(\gamma_{n}\right)
\end{array}\right] x, y\right] \\
& =\sum_{r=0}^{\infty} \sum_{s=0}^{\infty} \frac{\prod_{j=1}^{p}\left(a_{j}\right)_{r+s} \prod_{j=1}^{q}\left(b_{j}\right)_{r} \prod_{j=1}^{k}\left(c_{j}\right)_{s}}{\prod_{j=1}^{l}\left(\alpha_{j}\right)_{r+s} \prod_{j=1}^{m}\left(\beta_{j}\right)_{r} \prod_{j=1}^{n}\left(\gamma_{j}\right)_{s}} \frac{x^{r}}{r !} \frac{y^{s}}{s !} .
\end{aligned}
$$

Therefore after using (4.13), we finally get 


$$
\begin{aligned}
& g_{[r, s, n, m, k]}\left(y_{1}, y_{2}\right)=\frac{c^{2}(c+1)^{2} C_{s-1}}{(r-1) !(s-r-1) !(m+1)^{s-2}} \sum_{i=0}^{r-1} \sum_{j=0}^{s-r-1}(-1)^{i+j}\left(\begin{array}{c}
r-1 \\
i
\end{array}\right)\left(\begin{array}{c}
s-r-1 \\
j
\end{array}\right) \frac{1}{d g} \\
& \times \frac{\left(\alpha_{2} k_{2} y_{1}^{-\left(1+k_{2}\right)}\right)}{\left(\alpha_{2} y_{1}^{-k_{2}}\right)^{\beta}} \frac{\left(\alpha_{2} k_{2} y_{2}^{-\left(1+k_{2}\right)}\right)}{\left(\alpha_{2} y_{2}^{-k_{2}}\right)^{\beta}} F_{1: 1 ; 0}^{1: 2 ; 1}\left[\begin{array}{c}
(g) ; \quad(\beta) ; \quad(d) ;(\beta) ; \\
(g+1) ;(d+1) ;
\end{array} \quad \frac{-1}{\alpha_{2} y_{2}^{-k_{2}}, \frac{-1}{\alpha_{2} y_{1}^{-k_{2}}}}\right] .
\end{aligned}
$$

\section{Product Moments of two concomitants $Y_{[r, n, m, k]}$ and $Y_{[s, n, m, k]}$}

For Burr type III dstribution, the product Moments of two concomitants $Y_{[r, n, m, k]}$ and $Y_{[s, n, m, k]}$ is given as

$$
E\left(Y_{[r, n, m, k]}^{(a)} Y_{[s, n, m, k]}^{(b)}\right)=\int_{0}^{\infty} \int_{0}^{\infty} y_{1}^{a} y_{2}^{b} g_{[r, s, n, m, k]}\left(y_{1}, y_{2}\right) d y_{1} d y_{2}
$$

In view of (4.14) and (5.1), we have

$$
\begin{aligned}
E\left(Y_{[r, n, m, k]}^{(a)} Y_{[s, n, m, k]}^{(b)}\right)=A \int_{0}^{\infty} \int_{0}^{\infty} y_{1}^{a} y_{2}^{b} \frac{\left(\alpha_{2} k_{2} y_{1}^{-\left(1+k_{2}\right)}\right)}{\left(\alpha_{2} y_{1}^{-k_{2}}\right)^{\beta}} \frac{\left(\alpha_{2} k_{2} y_{2}^{-\left(1+k_{2}\right)}\right)}{\left(\alpha_{2} y_{2}^{-k_{2}}\right)^{\beta}} \\
\times F_{1: 1 ; 0}^{1: 2 ; 1}\left[\begin{array}{cc}
(g) ; & (\beta) ; \quad(d) ;(\beta) ; \\
(g+1) ;(d+1) ; & \left.; \frac{-1}{\alpha_{2} y_{2}^{-k_{2}}, \frac{-1}{\alpha_{2} y_{1}^{-k_{2}}}}\right] d y_{1} d y_{2}
\end{array}\right.
\end{aligned}
$$

where,

$$
A=\frac{c^{2}(c+1)^{2} C_{s-1}}{(r-1) !(s-r-1) !(m+1)^{s-2}} \sum_{i=0}^{r-1} \sum_{j=0}^{s-r-1}(-1)^{i+j}\left(\begin{array}{c}
r-1 \\
i
\end{array}\right)\left(\begin{array}{c}
s-r-1 \\
j
\end{array}\right) \frac{1}{d g}
$$

Thus,

$$
\begin{aligned}
& E\left(Y_{[r, n, m, k]}^{(a)} Y_{[s, n, m, k]}^{(b)}\right)=A \int_{0}^{\infty} \int_{0}^{\infty} y_{1}^{a} y_{2}^{b} \frac{\left(\alpha_{2} k_{2} y_{1}^{-\left(1+k_{2}\right)}\right)}{\left(\alpha_{2} y_{1}^{-k_{2}}\right)^{\beta}} \frac{\left(\alpha_{2} k_{2} y_{2}^{-\left(1+k_{2}\right)}\right)}{\left(\alpha_{2} y_{2}^{-k_{2}}\right)^{\beta}} \\
& \times \sum_{l=0}^{\infty} \sum_{p=0}^{\infty} \frac{(g)_{p+l}}{(g+1)_{p+l}} \frac{(\beta)_{l}(d)_{l}(\beta)_{p}}{(d+1)_{l}} \frac{\left(\frac{-1}{\alpha_{2} y_{1}^{-k_{2}}}\right)^{p}}{p !} \frac{\left(\frac{-1}{\alpha_{2} y_{2}^{-k_{2}}}\right)^{l}}{l !} d y_{1} d y_{2} .
\end{aligned}
$$

By Srivastava and Karlsson (1985), we have 


$$
(\lambda)_{m+n}=(\lambda)_{m}(\lambda+m)_{n}
$$

On applying (5.5) in (5.4), we get

$$
\begin{aligned}
& =A \int_{0}^{\infty} y_{2}^{b} \frac{\left(\alpha_{2} k_{2} y_{2}^{-\left(1+k_{2}\right)}\right)}{\left(\alpha_{2} y_{2}^{-k_{2}}\right)^{\beta}} \sum_{l=0}^{\infty} \frac{(g)_{l}}{(g+1)_{l}} \frac{(\beta)_{l}(d)_{l}}{(d+1)_{l}} \frac{\left(\frac{-1}{\alpha_{2} y_{2}^{-k_{2}}}\right)^{l}}{l !} \\
& \times\left\{\int_{0}^{\infty} y_{1}^{a} \frac{\left(\alpha_{2} k_{2} y_{1}^{-\left(1+k_{2}\right)}\right)}{\left(\alpha_{2} y_{1}^{-k_{2}}\right)^{\beta}} \sum_{p=0}^{\infty} \frac{(\beta)_{p}(g+l)_{p}}{(g+1+l)_{p}} \frac{\left(\frac{-1}{\left.\alpha_{2} y_{1}^{-k_{2}}\right)^{p}}\right.}{p !} d y_{1}\right\} d y_{2} \\
& =A \int_{0}^{\infty} y_{2}^{b} \frac{\left(\alpha_{2} k_{2} y_{2}^{-\left(1+k_{2}\right)}\right)}{\left(\alpha_{2} y_{2}^{-k_{2}}\right)^{\beta}} \sum_{l=0}^{\infty} \frac{(g)_{l}}{(g+1)_{l}} \frac{(\beta)_{l}(d)_{l}}{(d+1)_{l}} \frac{\left(\frac{-1}{\alpha_{2} y_{2}^{-k_{2}}}\right)^{l}}{l !} \\
& \quad \times \int_{0}^{\infty} y_{1}^{a} \frac{\left(\alpha_{2} k_{2} y_{1}^{-\left(1+k_{2}\right)}\right)}{\left(\alpha_{2} y_{1}^{-k_{2}}\right)^{\beta}}{ }_{2} F_{1}\left[\begin{array}{c}
(\beta) ; \quad(g+l) \\
(g+l+1)
\end{array}\right] d y_{1} d y_{2} .
\end{aligned}
$$

Now letting $\alpha_{2} y_{1}^{-k_{2}}=z-1$ in (5.7), we have

$$
\begin{aligned}
= & A\left(\alpha_{2}\right)^{\frac{a}{k_{2}}} \int_{0}^{\infty} y_{2} \frac{\left(\alpha_{2} k_{2} y_{2}^{-\left(1+k_{2}\right)}\right)}{\left(\alpha_{2} y_{2}^{-k_{2}}\right)^{\beta}} \sum_{l=0}^{\infty} \frac{(g)_{l}}{(g+1)_{l}} \frac{(\beta)_{l}(d)_{l}}{(d+1)_{l}} \frac{\left(\frac{-1}{\alpha_{2} y_{2}^{-k_{2}}}\right)^{l}}{l !} \\
& \times \int_{1}^{\infty}(z-1)^{-\left(\beta+\frac{a}{k_{2}}\right)}{ }_{2} F_{1}\left[\begin{array}{cc}
(\beta) ; & (g+l) \\
(g+l+1) & ; \frac{1}{1-z}
\end{array}\right] d z d y_{2}
\end{aligned}
$$

Since

$$
{ }_{2} F_{1}\left[\begin{array}{ll}
a, b & \\
& ; x
\end{array}\right]=(1-x)^{-a} 2 F_{1}\left[\begin{array}{ll}
a, c-b & \\
c & ;-\frac{x}{1-x}
\end{array}\right] .
$$

Therefore on using relation (5.9) in (5.10), we have

$$
\begin{aligned}
= & A\left(\alpha_{2}\right)^{\frac{a}{k_{2}}} \int_{0}^{\infty} y_{2}^{b} \frac{\left(\alpha_{2} k_{2} y_{2}^{-\left(1+k_{2}\right)}\right)}{\left(\alpha_{2} y_{2}^{-k_{2}}\right)^{\beta}} \sum_{l=0}^{\infty} \frac{(g)_{l}}{(g+1)_{l}} \frac{(\beta)_{l}(d)_{l}}{(d+1)_{l}} \frac{\left(\frac{-1}{\alpha_{2} y_{2}^{-k_{2}}}\right)^{l}}{l !} \\
& \times \sum_{p=0}^{\infty} \frac{(\beta)_{p}(1)_{p}}{(g+l+1)_{p}}\left\{\int_{1}^{\infty}(z-1)^{\left(1-\frac{a}{k_{2}}\right)-1} z^{-(\beta+p)} d z\right\} d y_{2} .
\end{aligned}
$$


Now using relation (3.5) in (5.10), we get

$$
\begin{aligned}
= & A\left(\alpha_{2}\right)^{\frac{a}{k_{2}}} \frac{g}{(g+l)} \frac{\Gamma\left(\beta-1+\frac{a}{k_{2}}\right) \Gamma\left(1-\frac{a}{k_{2}}\right)}{\Gamma(\beta)} \int_{0}^{\infty} y_{2}^{b} \frac{\left(\alpha_{2} k_{2} y_{2}^{-\left(1+k_{2}\right)}\right)}{\left(\alpha_{2} y_{2}^{-k_{2}}\right)^{\beta}} \\
& \left.\times \sum_{l=0}^{\infty} \frac{(\beta)_{l}(d)_{l}}{(d+1)_{l}} \frac{\left(\frac{-1}{\left.\alpha_{2} y_{2}^{-k_{2}}\right)^{l}}\right.}{l !}{ }_{2} F_{1}\left[\begin{array}{c}
\left(\beta-1+\frac{a}{k_{2}}\right) ; 1 \\
(g+l+1)
\end{array}\right] 1\right] d y_{2} .
\end{aligned}
$$

Now in view of (3.14), (5.11) becomes

$$
\begin{aligned}
& =\left(\alpha_{2}\right)^{\frac{a}{k_{2}}} g A \frac{\Gamma\left(\beta-1+\frac{a}{k_{2}}\right) \Gamma\left(1-\frac{a}{k_{2}}\right)}{\Gamma(\beta)}\left\{\int_{0}^{\infty} y_{2}^{b} \frac{\left(\alpha_{2} k_{2} y_{2}^{-\left(1+k_{2}\right)}\right)}{\left(\alpha_{2} y_{2}^{-k_{2}}\right)^{\beta}} d y_{2}\right\} \\
& \times \sum_{l=0}^{\infty} \frac{(\beta)_{l}(d)_{l}}{(d+1)_{l}} \frac{\left(\frac{-1}{\alpha_{2} y_{2}^{-k_{2}}}\right)^{l}}{l !} \frac{1}{\left(g+1-\beta-\frac{a}{k_{2}}+l\right)} .
\end{aligned}
$$

Using relation (4.10) in (5.12), we get

$$
\begin{aligned}
& =\left(\alpha_{2}\right)^{\frac{a}{k_{2}}} A \frac{g}{\left(g+1-\beta-\frac{a}{k_{2}}\right)} \frac{\Gamma\left(\beta-1+\frac{a}{k_{2}}\right) \Gamma\left(1-\frac{a}{k_{2}}\right)}{\Gamma(\beta)}\left\{\int_{0}^{\infty} y_{2}^{b} \frac{\left(\alpha_{2} k_{2} y_{2}^{-\left(1+k_{2}\right)}\right)}{\left(\alpha_{2} y_{2}^{-k_{2}}\right)^{\beta}} d y_{2}\right\} \\
& \times \sum_{l=0}^{\infty} \frac{(\beta)_{l}(d)_{l}}{(d+1)_{l}} \frac{\left(g+1-\beta-\frac{a}{k_{2}}\right)_{l}}{\left(g+2-\beta-\frac{a}{k_{2}}\right)_{l}} \frac{\left(\frac{-1}{\alpha_{2} y_{2}^{-k_{2}}}\right)^{l}}{l !} .
\end{aligned}
$$

Further setting $z-1=\alpha_{2} y_{2}^{-k_{2}}$ in (5.13), it becomes

$$
\begin{aligned}
=\left(\alpha_{2}\right)^{\frac{(a+b)}{k_{2}}} A \frac{g}{\left(g+1-\beta-\frac{a}{k_{2}}\right)} \frac{\Gamma\left(\beta-1+\frac{a}{k_{2}}\right) \Gamma\left(1-\frac{a}{k_{2}}\right)}{\Gamma(\beta)} \\
\times \int_{1}^{\infty}(z-1)^{-\left(\beta+\frac{b}{k_{2}}\right)}{ }_{3} F_{2}\left[\begin{array}{c}
(d) ;\left(g+1-\beta-\frac{a}{k_{2}}\right) ;(\beta) \\
(d+1) ;\left(g+2-\beta-\frac{a}{k_{2}}\right)
\end{array} \quad \frac{1}{1-z}\right] d z .
\end{aligned}
$$

Set $z-1=\frac{1}{t}$ in (5.14), to get

$$
=\left(\alpha_{2}\right)^{\frac{(a+b)}{k_{2}}} A \frac{g}{\left(g+1-\beta-\frac{a}{k_{2}}\right)} \frac{\Gamma\left(\beta-1+\frac{a}{k_{2}}\right) \Gamma\left(1-\frac{a}{k_{2}}\right)}{\Gamma(\beta)}
$$




$$
\times \int_{0}^{\infty} t^{\left(\beta+\frac{b}{k_{2}}-1\right)-1}{ }_{3} F_{2}\left[\begin{array}{cc}
(d) ; & \left(g+1-\beta-\frac{a}{k_{2}}\right) ; \beta \\
(d+1) ; & \left(g+2-\beta-\frac{a}{k_{2}}\right)
\end{array}\right] d z .
$$

Note that [Prudnikov et al., 1986]

$$
\begin{aligned}
& \int_{0}^{\infty} x^{s-1}{ }_{3} F_{2}\left[\begin{array}{c}
\left(a_{1}\right),\left(a_{2}\right),\left(a_{3}\right) \\
\left(b_{1}\right),\left(b_{2}\right)
\end{array} ;-x\right] d x \\
& =\frac{\Gamma\left(b_{1}\right) \Gamma\left(b_{2}\right) \Gamma(s) \Gamma\left(a_{1}-s\right) \Gamma\left(a_{2}-s\right) \Gamma\left(a_{3}-s\right)}{\Gamma\left(a_{1}\right) \Gamma\left(a_{2}\right) \Gamma\left(a_{3}\right) \Gamma\left(b_{1}-s\right) \Gamma\left(b_{2}-s\right)}
\end{aligned}
$$

Now using relations (5.16) in (5.15), we get

$$
\begin{aligned}
& =\left(\alpha_{2}\right)^{\frac{(a+b)}{k_{2}}} A \frac{g}{\left(g+2-2 \beta-\frac{a}{k_{2}}-\frac{b}{k_{2}}\right)\left(d+1-\beta-\frac{b}{k_{2}}\right)} \frac{\Gamma\left(\beta-1+\frac{a}{k_{2}}\right) \Gamma\left(1-\frac{a}{k_{2}}\right)}{\Gamma(\beta)} \\
& \times \frac{\Gamma\left(\beta-1+\frac{b}{k_{2}}\right) \Gamma\left(1-\frac{b}{k_{2}}\right)}{\Gamma(\beta)} .
\end{aligned}
$$

Finally putting the values of $A, d, g$ and $\beta$ in (5.17), we have

$$
\begin{aligned}
& E\left(Y_{[r, n, m, k]}^{(a)} Y_{[s, n, m, k]}^{(b)}\right)=\left(\alpha_{2}\right)^{\frac{(a+b)}{k_{2}}} \frac{c^{2}(c+1)^{2} C_{s-1}}{(r-1) !(s-r-1) !(m+1)^{s-2}} \frac{\Gamma\left(\beta-1+\frac{a}{k_{2}}\right) \Gamma\left(1-\frac{a}{k_{2}}\right)}{\Gamma(\beta)} \\
& \times \frac{\Gamma\left(\beta-1+\frac{b}{k_{2}}\right) \Gamma\left(1-\frac{b}{k_{2}}\right)}{\Gamma(\beta)} \sum_{i=0}^{r-1} \sum_{j=0}^{s-r-1}(-1)^{i+j}\left(\begin{array}{c}
r-1 \\
i
\end{array}\right)\left(\begin{array}{c}
s-r-1 \\
j
\end{array}\right) \\
& \times \frac{1}{\left[c\{k+(n-s+j)(m+1)\}+\frac{b}{k_{2}}\right]} \frac{1}{\left[c\{k+(n-r+i)(m+1)\}+\frac{a}{k_{2}}+\frac{b}{k_{2}}\right]} \\
& =\left(\alpha_{2}\right)^{\frac{(a+b)}{k_{2}}} \frac{c^{2}(c+1)^{2} C_{s-1}}{(r-1) !(s-r-1) !(m+1)^{s}} \frac{\Gamma\left(\beta-1+\frac{a}{k_{2}}\right) \Gamma\left(1-\frac{a}{k_{2}}\right)}{\Gamma(\beta)} \\
& \times \frac{\Gamma\left(\beta-1+\frac{b}{k_{2}}\right) \Gamma\left(1-\frac{b}{k_{2}}\right)}{\Gamma(\beta)} \sum_{i=0}^{r-1}(-1)^{i}\left(\begin{array}{c}
r-1 \\
i
\end{array}\right) B\left(\frac{k}{m+1}+(n-r+i)+\frac{a+b}{c k_{2}(m+1)}, 1\right) \\
& \times \sum_{j=0}^{s-r-1}(-1)^{j}\left(\begin{array}{c}
s-r-1 \\
j
\end{array}\right) B\left(\frac{k}{m+1}+(n-s+j)+\frac{b}{c k_{2}(m+1)}, 1\right) .
\end{aligned}
$$


On using relation (3.17) in (5.19), we get

$$
\begin{aligned}
= & \left(\alpha_{2}\right)^{\frac{(a+b)}{k_{2}}} \frac{(c+1)^{2} C_{s-1}}{(r-1) !(s-r-1) !(m+1)^{s}} \frac{\Gamma\left(\beta-1+\frac{a}{k_{2}}\right) \Gamma\left(1-\frac{a}{k_{2}}\right)}{\Gamma(\beta)} \\
& \times \frac{\Gamma\left(\beta-1+\frac{b}{k_{2}}\right) \Gamma\left(1-\frac{b}{k_{2}}\right)}{\Gamma(\beta)} B\left(\frac{k}{m+1}+(n-r)+\frac{a+b}{c k_{2}(m+1)}, r\right) \\
& \times B\left(\frac{k}{m+1}+(n-s)+\frac{b}{c k_{2}(m+1)}, s-r\right),
\end{aligned}
$$

which after simplification yields

$$
\begin{aligned}
E\left(Y_{[r, n, m, k]}^{(a)} Y_{[s, n, m, k]}^{(b)}\right)=\left(\alpha_{2}\right)^{\frac{(a+b)}{k_{2}}}(c+1)^{2} \frac{\Gamma\left(\beta-1+\frac{a}{k_{2}}\right) \Gamma\left(1-\frac{a}{k_{2}}\right)}{\Gamma(\beta)} \\
\times \frac{\Gamma\left(\beta-1+\frac{b}{k_{2}}\right) \Gamma\left(1-\frac{b}{k_{2}}\right)}{\Gamma(\beta)} \frac{1}{\prod_{i=1}^{r}\left(1+\frac{a+b}{c k_{2} \gamma_{i}}\right) \prod_{j=r+1}^{s}\left(1+\frac{b}{c k_{2} \gamma_{i}}\right)} .
\end{aligned}
$$

Remark 5.1 : By setting $m=0, k=1$ in (5.18), we get product moments of concomitant of order statistics from bivariate Burr III distribution as

$$
\begin{aligned}
E\left(Y_{[n-s+1: n]}^{(a)} Y_{[n-r+1: n]}^{(b)}\right)= & \left(\alpha_{2}\right)^{\frac{(a+b)}{k_{2}}} \frac{c^{2}(c+1)^{2} n !}{(r-1) !(s-r-1) !(n-s) !} \frac{\Gamma\left(\beta-1+\frac{a}{k_{2}}\right) \Gamma\left(1-\frac{a}{k_{2}}\right)}{\Gamma(\beta)} . \\
& \times \frac{\Gamma\left(\beta-1+\frac{b}{k_{2}}\right) \Gamma\left(1-\frac{b}{k_{2}}\right)}{\Gamma(\beta)} \sum_{i=0}^{r-1} \sum_{j=0}^{s-r-1}(-1)^{i+j}\left(\begin{array}{c}
r-1 \\
i
\end{array}\right)\left(\begin{array}{c}
s-r-1 \\
j
\end{array}\right) \\
\times & \frac{1}{\left[c(n-s+1+j)+\frac{b}{k_{2}}\right]} \frac{1}{\left[c(n-r+1+i)+\frac{a}{k_{2}}+\frac{b}{k_{2}}\right]}
\end{aligned}
$$

Replace $n-s+1$ by $r$ and $n-r+1$ by $s$ in (5.22), we get

$$
\begin{aligned}
& E\left(Y_{[r: n]}^{(a)} Y_{[s: n]}^{(b)}\right)=\left(\alpha_{2}\right)^{\frac{(a+b)}{k_{2}}} \frac{c^{2}(c+1)^{2} n !}{(r-1) !(s-r-1) !(n-s) !} \frac{\Gamma\left(\beta-1+\frac{a}{k_{2}}\right) \Gamma\left(1-\frac{a}{k_{2}}\right)}{\Gamma(\beta)} \\
& \times \frac{\Gamma\left(\beta-1+\frac{b}{k_{2}}\right) \Gamma\left(1-\frac{b}{k_{2}}\right)}{\Gamma(\beta)} \sum_{i=0}^{n-s} \sum_{j=0}^{s-r-1}(-1)^{i+j}\left(\begin{array}{c}
n-s \\
i
\end{array}\right)\left(\begin{array}{c}
s-r-1 \\
j
\end{array}\right) \\
& \times \frac{1}{\left[c r+c j+\frac{b}{k_{2}}\right]} \frac{1}{\left[c s+i c+\frac{a}{k_{2}}+\frac{b}{k_{2}}\right]}
\end{aligned}
$$




$$
\begin{aligned}
E\left(Y_{[r: n]}^{(a)} Y_{[s: n]}^{(b)}\right) & =\frac{n !}{(r-1) !}\left(\alpha_{2}\right)^{\frac{(a+b)}{k_{2}}} \frac{\Gamma\left(c+1+\frac{a}{k_{2}}\right) \Gamma\left(1-\frac{a}{k_{2}}\right) \Gamma\left(c+1+\frac{b}{k_{2}}\right) \Gamma\left(1-\frac{b}{k_{2}}\right)}{\Gamma(c+1)} \\
& \times \frac{\Gamma\left(r+\frac{b}{c k_{2}}\right)}{\Gamma\left(s+\frac{b}{c k_{2}}\right)} \frac{\Gamma\left(s+\frac{a+b}{c k_{2}}\right)}{\Gamma\left(n+1+\frac{a+b}{c k_{2}}\right)}
\end{aligned}
$$

as obtained by (Begum and Khan, 1998).

Remark 5.2 : If $m=-1$ in (5.21), we get product moment of concomitants of $k$-th lower record values from bivariate Burr III distribution as

$$
\begin{aligned}
E\left(Y_{[r, n,-1, k]}^{(a)} Y_{[s, n,-1, k]}^{(b)}\right) & =\left(\alpha_{2}\right)^{\frac{(a+b)}{k_{2}}}(c+1)^{2} \frac{\Gamma\left(\beta-1+\frac{a}{k_{2}}\right) \Gamma\left(1-\frac{a}{k_{2}}\right)}{\Gamma(\beta)} \\
& \times \frac{\Gamma\left(\beta-1+\frac{b}{k_{2}}\right) \Gamma\left(1-\frac{b}{k_{2}}\right)}{\Gamma(\beta)} \frac{1}{\left(1+\frac{a+b}{c k k_{2}}\right)^{r}\left(1+\frac{b}{c k k_{2}}\right)^{s-r}} .
\end{aligned}
$$

Putting the value of $\beta$ in (5.22), we get

$$
\begin{aligned}
E\left(Y_{[r, n,-1, k]}^{(a)} Y_{[s, n,-1, k]}^{(b)}\right)=\left(\alpha_{2}\right)^{\frac{(a+b)}{k_{2}}} \frac{\Gamma\left(c+1+\frac{a}{k_{2}}\right) \Gamma\left(1-\frac{a}{k_{2}}\right)}{\Gamma(c+1)} \\
\times \frac{\Gamma\left(c+1+\frac{b}{k_{2}}\right) \Gamma\left(1-\frac{b}{k_{2}}\right)}{\Gamma(c+1)} \frac{1}{\left(1+\frac{a+b}{c k k_{2}}\right)^{r}\left(1+\frac{b}{c k k_{2}}\right)^{s-r}}
\end{aligned}
$$

\section{Acknowledgement}

The authors are thankful to Professor M. Ahsanullah, Rider University, Lawrenceville, NJ, USA for his fruitful suggestions.

\section{References}

[1] Ahsanullah, M. (2004): Record Values-Theory and Applications. University Press of America, Lanham, Maryland.

[2] Begum, A.A. and Khan, A.H. (1998): Concomitants of order statistics from bivariates Burr III distribution. Journal of Statistical Researh, 32(2), 15-25.

[3] Burkschat, M.; Cramer, E. and Kamps, U. (2003): Dual generalized order statistics. Metron, LXI(1), 13 - 26.

[4] David, H. A. and Nagaraja, H. N. (2003): Order Statistics, John Wiley, New York.

[5] Erdélyi, A., Magnus, W., Oberhetinger, F. and Tricomi, F. G.(1954): Tables of Integral Transforms. McGraw-Hill, New York.

[6] Kamps, U. (1995): A concept of generalized order statistics. B.G. Teubner Stuttgart, Germany.

[7] Prudnikov, A. P., Brychkov, Yu. A. and Marichev, O. I. (1986): Integral and series: More Special Functions (Vol.3). Gordon and Breach Science Publisher, New York. 
Concomitants of Dual Generalized Order Statistics ...

[8] Rodriguez, R. N. (1982): Burr distributions: Encyclopedia of Statistical Science. Vol. 1, (Kotz and Johnson, ed.), 335-340. John Wiley and Sons, New York.

[9] Srivastava, H. M. and Karlsson (1985): Multiple Gaussian Hypergeometric series. John Wiley \& Sons, New York. 